Preprint JINR E2-94-443, Dubna 1994

hep-th/9508129

\title{
Some new integrable equations from the self-dual Yang-Mills equations
}

\author{
T.A.Ivanova円 and A.D.Popov尹 \\ Bogoliubov Laboratory of Theoretical Physics, \\ JINR, Dubna 141980, Moscow Region, Russia
}

\begin{abstract}
.
Using the symmetry reductions of the self-dual Yang-Mills (SDYM) equations in $(2+2)$ dimensions, we introduce new integrable equations which are nonautonomous versions of the chiral model in $(2+1)$ dimensions, generalized nonlinear Schrödinger, Korteweg-de Vries, Toda lattice, Garnier and Euler-Arnold equations. The Lax pairs for all of these equations are derived by the symmetry reductions of the Lax pair for the SDYM equations.
\end{abstract}

\footnotetext{
${ }^{1}$ e-mail: ita@thsun1.jinr.dubna.su

${ }^{2}$ e-mail: popov@thsun1.jinr.dubna.su
} 


\section{Introduction}

The purpose of this paper is to describe six new systems of differential equations and to write out the Lax pairs for them. We derive equations for all these integrable systems using the method of symmetry reduction (see [1,2] and references therein) applied to the self-dual Yang-Mills (SDYM) equations in the space $R^{2,2}$ with the metric of the signature $(++--)$. For derivation of the Lax pairs for these equations we use the algorithm of reduction of the Lax pair for the SDYM equations described in [3].

We use the SDYM equations in $R^{2,2}$ and the symmetry reduction method only as a tool for obtaining new integrable systems in lower dimensions, but there are at least three reasons in view of which the connection between these integrable systems and the SDYM equations is important. Firstly, the importance of the SDYM equations in $R^{2,2}$ is motivated by the conjecture [4] that the SDYM equations may be a universal integrable system, i.e. that all integrable equations in $1 \leq d \leq 3$ dimensions can be obtained from it by suitable reductions. In fact, it has recently been shown that many integrable equations can be embedded into the SDYM equations [4-14]. It is obvious that besides the known equations, the symmetry reductions of the SDYM equations give the opportunity to obtain some new integrable equations valuable for applications [3]. In the following, we illustrate this by deriving nonautonomous versions of the equations mentioned in the abstract. Secondly, to the equations derived from the SDYM equations, one may apply the twistor techniques for solving equations and for analysing properties of solutions (see, e.g., $[15,16,10,11])$. Thirdly, the SDYM equations are known to arise in the $N=2$ supersymmetric string theory $[17,18]$ which is considered now as the universal string theory including the conventional $N=0$ and $N=1$ strings as particular vacua [19, 20]. Therefore, the soliton-type solutions of the SDYM equations and their reductions are important for the analysis of nonperturbative effects in string theories.

\section{Definitions and notation}

We consider the space $R^{2,2}$ with the metric $\left(g_{\mu \nu}\right)=\operatorname{diag}(+1,+1,-1,-1)$ and the potentials $A_{\mu}$ of the Yang-Mills (YM) fields $F_{\mu \nu}=\partial_{\mu} A_{\nu}-\partial_{\nu} A_{\mu}+\left[A_{\mu}, A_{\nu}\right]$, where $\mu, \nu, \ldots=$ $1, \ldots, 4, \partial_{\mu}=\partial / \partial x^{\mu}$. Fields $A_{\mu}$ and $F_{\mu \nu}$ take values in the Lie algebra $g l(n, C)$.

In $R^{2,2}$ we introduce null coordinates $t=\frac{1}{2}\left(x^{2}-x^{4}\right), u=\frac{1}{2}\left(x^{2}+x^{4}\right), y=\frac{1}{2}\left(x^{1}-x^{3}\right), z=$ $\frac{1}{2}\left(x^{1}+x^{3}\right)$ and set $A_{t}=A_{2}-A_{4}, A_{u}=A_{2}+A_{4}, A_{y}=A_{1}-A_{3}, A_{z}=A_{1}+A_{3}$. The 
SDYM equations in the null coordinates have the following form:

$$
F_{t z}=0, \quad F_{u y}=0, \quad F_{t u}+F_{z y}=0
$$

Equations (1) can be obtained as compatibility conditions of the following linear system of equations (cf. ref. [21, 22]):

$$
\begin{gathered}
\left(\partial_{t}-\lambda \partial_{y}+A_{t}-\lambda A_{y}\right) \Psi=0, \\
\left(\partial_{z}+\lambda \partial_{u}+A_{z}+\lambda A_{u}\right) \Psi=0, \\
\partial_{\bar{\lambda}} \Psi=0,
\end{gathered}
$$

where $\bar{\lambda}$ is a complex conjugate to $\lambda$. Here $\Psi$ is a column vector depending on the coordinates of $R^{2,2}$ and the "coordinates" $\lambda, \bar{\lambda}$, parametrizing the upper sheet of the hyperboloid $H^{2}=S O(2,1) / S O(2)$. Notice that $\Psi$ is defined on the twistor space $\mathcal{Z}=$ $R^{2,2} \times H^{2}$ for the space $R^{2,2}$, and eqs.(2) mean the holomorphicity of the vector-function $\Psi$ (Ward theorem $[22,15])$.

\section{Symmetry reduction}

We consider the inhomogeneous group of rotations $I S O(2,2)$ (rotations and translations) and an arbitrary subgroup $G$ of the group $\operatorname{ISO}(2,2)$. We would like to impose the conditions of $G$-invariance on the YM potentials $A_{\mu}$ and on the vector-function $\Psi$. For that, we have to define the generators of the group $I S O(2,2)$ as vector fields on $R^{2,2}$ when considering the action of $G$ on $A_{\mu}$, and as vector fields on the twistor space $R^{2,2} \times H^{2}$ when considering the action of $G$ on $\Psi[3]$.

Let us introduce the following constant tensors:

$$
\begin{aligned}
& f_{\mu \nu}^{a}=\left\{f_{b c}^{a}, \mu=a, \nu=b ; \delta_{\mu}^{a}, \nu=4 ;-\delta_{\nu}^{a}, \mu=4\right\}, \quad I_{a}{ }_{\nu}^{\mu}=-\frac{1}{2} g_{a b} g^{\mu \lambda} f_{\lambda \nu}^{b}, \\
& \bar{f}_{\mu \nu}^{a}=\left\{f_{b c}^{a}, \mu=a, \nu=b ;-\delta_{\mu}^{a}, \nu=4 ; \delta_{\nu}^{a}, \mu=4\right\}, \quad J_{a}{ }_{\nu}^{\mu}=-\frac{1}{2} g_{a b} g^{\mu \lambda} \bar{f}_{\lambda \nu}^{b},
\end{aligned}
$$

where $a, b, \ldots=1,2,3, g_{11}=g_{22}=-g_{33}=1$ and $f_{23}^{1}=f_{31}^{2}=-f_{12}^{3}=1$ are the structure constants of the group $S O(2,1)$. Then, the generators of the group $I S O(2,2)$ can be realized in terms of the following vector fields on $R^{2,2}$ :

$$
X_{a}=I_{a}{ }_{\nu}^{\mu} x^{\nu} \partial_{\mu}, \quad Y_{a}=J_{a}{ }_{\nu}^{\mu} x^{\nu} \partial_{\mu}, \quad P_{\mu}=\partial_{\mu}
$$

The vector fields on $\mathcal{Z}=R^{2,2} \times H^{2}$, which also form the generators of $I S O(2,2)$, are given by

$$
\tilde{X}_{a}=X_{a}, \quad \tilde{Y}_{a}=Y_{a}+Z_{a}, \quad \widetilde{P}_{\mu}=P_{\mu}
$$


with the following expression of the generators $Z_{a}$ of the $S O(2,1)$-rotations on $H^{2}$ :

$$
Z_{1}=\frac{1}{2}\left[\left(1-\lambda^{2}\right) \partial_{\lambda}+\left(1-\bar{\lambda}^{2}\right) \partial_{\bar{\lambda}}\right], Z_{2}=-\left[\lambda \partial_{\lambda}+\bar{\lambda} \partial_{\bar{\lambda}}\right], Z_{3}=-\frac{1}{2}\left[\left(1+\lambda^{2}\right) \partial_{\lambda}+\left(1+\bar{\lambda}^{2}\right) \partial_{\bar{\lambda}}\right] .
$$

It can be easily shown that $\left[X_{a}, X_{b}\right]=f_{a b}^{c} X_{c},\left[Z_{a}, Z_{b}\right]=f_{a b}^{c} Z_{c},\left[\tilde{Y}_{a}, \tilde{Y}_{b}\right]=f_{a b}^{c} \tilde{Y}_{c}$ and so on.

In order to reduce the SDYM equations (1) and the linear system (2) under a subgroup $G$ of the group $I S O(2,2)$, it is necessary to impose the following conditions of $G$-invariance on the gauge potentials $A_{\mu}$ and on the vector-function $\Psi$ [23]:

$$
\begin{gathered}
W_{\xi} A_{\mu}+A_{\sigma} W_{\xi, \mu}^{\sigma}=0, \quad \forall \xi \in \mathcal{G}, \\
\tilde{W}_{\xi} \Psi=0, \quad \forall \xi \in \mathcal{G},
\end{gathered}
$$

where $\mathcal{G}$ is a Lie algebra of the group $G, W_{\xi}=W_{\xi}^{\sigma} \partial_{\sigma}$ are vector fields on $R^{2,2}$ and $\tilde{W}_{\xi}=\tilde{W}_{\xi}^{\sigma} \partial_{\sigma}+\tilde{W}_{\xi}^{a} Z_{a}$ are vector fields on $R^{2,2} \times H^{2}$. Both $W_{\xi}$ and $\tilde{W}_{\xi}$ form a realization of the Lie algebra $\mathcal{G}$.

In accordance with the general method of symmetry reduction (see [1] and references therein), as new coordinates on $R^{2,2} \times H^{2}$, one should choose the coordinates $\theta_{\xi}$ on the orbits $Q$ of the group $G$ in $R^{2,2} \times H^{2}$, and the invariant coordinates $\theta_{A}(A=1, \ldots, 4-\operatorname{dim} Q)$ and $\zeta$ which parametrize the space of orbits and satisfy

$$
\tilde{W}_{\xi} \theta_{A}=0, \quad \tilde{W}_{\xi} \zeta=0, \quad \partial_{\bar{\lambda}} \zeta=0, \quad \forall \xi \in \mathcal{G}
$$

Here, the invariant complex coordinate $\zeta$ represents the new "spectral parameter". Then, substituting solutions of eqs.(6) and (7) into eqs.(1), (2), we obtain the reduced SDYM equations and their Lax pairs in terms of functions of the invariant coordinates $[1,3]$.

Now we consider examples of reduction of the SDYM equations to the integrable equations in $1 \leq d \leq 3$. In what follows, we shall firstly write out some known integrable equations which arise as reduction of the SDYM equations under translations. After that we shall describe new nonautonomous versions of these equations, derived via reduction with respect to the action of the subgroups containing rotations.

\section{Reductions to integrable systems in $(2+1)$ dimensions}

Chiral model equation in $R^{2,1}[24,16]$. Let us consider the one-dimensional Abelian group with generator $P_{y}-P_{z}$. Then, $\varphi=y-z$ will be the coordinate on the orbit and the invariant coordinates are $x=y+z, t, u$ and $\lambda$. The YM potentials $A_{\mu}$, satisfying (6a), and the vector-function $\Psi$, satisfying (6b) and (2c), are given by

$$
A_{t}=T_{t}(t, u, x), A_{u}=T_{u}(t, u, x), A_{y}=T_{y}(t, u, x), A_{z}=T_{z}(t, u, x), \Psi=\psi(t, u, x, \lambda) .
$$


Substituting (8) into the linear system (2), we obtain the following reduced Lax pair:

$$
\left(\partial_{t}-\lambda \partial_{x}+T_{t}-\lambda T_{y}\right) \psi=0, \quad\left(\partial_{x}+\lambda \partial_{u}+T_{z}+\lambda T_{u}\right) \psi=0
$$

Accordingly, the SDYM equations (1) are reduced to the compatibility conditions of the Lax pair (9):

$$
\begin{gathered}
\partial_{t} T_{z}-\partial_{x} T_{t}+\left[T_{t}, T_{z}\right]=0, \quad \partial_{x} T_{u}-\partial_{u} T_{y}+\left[T_{y}, T_{u}\right]=0 \\
\partial_{x}\left(T_{y}-T_{z}\right)+\partial_{t} T_{u}-\partial_{u} T_{t}+\left[T_{t}, T_{u}\right]+\left[T_{z}, T_{y}\right]=0 .
\end{gathered}
$$

Now let us choose the gauge $T_{z}=T_{t}=0$. Then, from eqs.(10a) we obtain $T_{u}=$ $g^{-1} \partial_{u} g, T_{y}=g^{-1} \partial_{x} g$, where $g$ is an arbitrary function of $t, u, x$ with values in the group $G L(n, C)$, and eqs. (10b) coincide with the equations of the chiral field model considered in the papers $[24,16]$ :

$$
\partial_{x}\left(g^{-1} \partial_{x} g\right)+\partial_{t}\left(g^{-1} \partial_{u} g\right)=0
$$

Nonautonomous chiral model equation in $R^{2,1}$. Now we consider the one-dimensional Abelian group of rotations generated by the vector field $X_{2}+Y_{2}$. From (4) and (5), we obtain $\tilde{X}_{2}+\tilde{Y}_{2}=X_{2}+Y_{2}+Z_{2}=z \partial_{z}-y \partial_{y}-\lambda \partial_{\lambda}-\bar{\lambda} \partial_{\bar{\lambda}}$. Let us introduce the coordinates $\rho, \theta, \eta, \xi$ by formulae $y=\frac{1}{2} \rho e^{-\theta}, z=\frac{1}{2} \rho e^{\theta}, \lambda=\eta e^{i \xi}$, then $X_{2}+Y_{2}=\partial_{\theta}$ and $\tilde{X}_{2}+\tilde{Y}_{2}=\partial_{\theta}-\eta \partial_{\eta}$. Therefore, $\varphi=\frac{1}{2}(\theta-\ln \eta)$ will be the coordinate on the orbit and $t, u, \rho, \zeta=\lambda e^{\theta}$ will be the invariant coordinates.

The invariant YM potentials $A_{\mu}$, satisfying eqs.(6a), have the form

$$
A_{t}=T_{t}(t, u, \rho), A_{u}=T_{u}(t, u, \rho), A_{y}=T_{y}(t, u, \rho) e^{\theta}, A_{z}=T_{z}(t, u, \rho) e^{-\theta}
$$

The vector-function

$$
\Psi=\psi(t, u, \rho, \zeta)
$$

is the solution of equations $(6 \mathrm{~b})$ and $(2 \mathrm{c})$.

Substituting (12) into (2), we obtain the following reduced Lax pair:

$$
\nabla_{V_{1}} \psi \equiv\left[\partial_{t}-\zeta \partial_{\rho}+\frac{1}{\rho} \zeta^{2} \partial_{\zeta}+T_{t}-\zeta T_{y}\right] \psi=0, \nabla_{V_{2}} \psi \equiv\left[\partial_{\rho}+\zeta \partial_{u}+\frac{1}{\rho} \zeta \partial_{\zeta}+T_{z}+\zeta T_{u}\right] \psi=0
$$

where $V_{1}=\partial_{t}-\zeta \partial_{\rho}+\frac{1}{\rho} \zeta^{2} \partial_{\zeta}, V_{2}=\partial_{\rho}+\zeta \partial_{u}+\frac{1}{\rho} \zeta \partial_{\zeta}$. Remind that in the general case $\left[V_{1}, V_{2}\right] \neq 0$ and then for linear systems like (13) the compatibility condition is

$$
\left[\nabla_{V_{1}}, \nabla_{V_{2}}\right]-\nabla_{\left[V_{1}, V_{2}\right]}=0
$$


Correspondingly, the SDYM equations (1) are reduced to

$$
\begin{gathered}
\partial_{t} T_{z}-\partial_{\rho} T_{t}+\left[T_{t}, T_{z}\right]=0, \quad \partial_{\rho} T_{u}-\partial_{u} T_{y}+\left[T_{y}, T_{u}\right]=0, \\
\partial_{\rho}\left(T_{y}-T_{z}\right)+\frac{1}{\rho}\left(T_{y}-T_{z}\right)+\partial_{t} T_{u}-\partial_{u} T_{t}+\left[T_{t}, T_{u}\right]+\left[T_{z}, T_{y}\right]=0
\end{gathered}
$$

which agree with the compatibility condition (14) of the Lax pair (13).

Choosing the same gauge $T_{z}=T_{t}=0$ as in (9), (10), from eqs.(15a) we obtain $T_{u}=g^{-1} \partial_{u} g, T_{y}=g^{-1} \partial_{\rho} g$. Then, eq. $(15 \mathrm{~b})$ is reduced to the nonautonomous chiral model equation in $R^{2,1}$ :

$$
\partial_{\rho}\left(g^{-1} \partial_{\rho} g\right)+\frac{1}{\rho} g^{-1} \partial_{\rho} g+\partial_{t}\left(g^{-1} \partial_{u} g\right)=0 \Longleftrightarrow \frac{1}{\rho} \partial_{\rho}\left(\rho g^{-1} \partial_{\rho} g\right)+\partial_{t}\left(g^{-1} \partial_{u} g\right)=0 .
$$

The Lax pair for this equation has the form (13) with $T_{z}=T_{t}=0$.

Remark. Notice that if one uses an additional condition of invariance under $P_{t}+P_{u}$ : $\left(\partial_{t}+\partial_{u}\right) \psi=\left(\partial_{t}+\partial_{u}\right) g=0$ in the Lax pair (9) and in eqs.(11), then one obtains the equation of the principal chiral model in $R^{1,1}$. But if we impose the same condition on $\psi$ and $g$ in (13) and (16), then we obtain the nonautonomous equation of the principal chiral model in $R^{1,1}[25,26]$, which in a particular case of the gauge group $G L(2, R)$ is equivalent to the Ernst equations [25, 10].

\section{Reductions to integrable systems in $(1+1)$ dimensions}

Generalized nonlinear Schrödinger equation (NLS) [9]. Let us consider the twodimensional Abelian group with the generators $\left\{P_{y}-P_{z}, P_{u}\right\}$. Then, solutions $A_{\mu}$ and $\Psi$ of eqs.(6) and (2c) are given by

$$
A_{t}=T_{t}(t, x), A_{u}=T_{u}(t, x), A_{y}=T_{y}(t, x), A_{z}=T_{z}(t, x), \Psi=\psi(t, x, \lambda) .
$$

The linear system (2) is reduced to the following one:

$$
\left\{\begin{array} { c } 
{ ( \partial _ { t } - \lambda \partial _ { x } + T _ { t } - \lambda T _ { y } ) \psi = 0 , } \\
{ ( \partial _ { x } + T _ { z } + \lambda T _ { u } ) \psi = 0 }
\end{array} \Rightarrow \left\{\begin{array}{c}
{\left[\partial_{t}+T_{t}+\lambda\left(T_{z}-T_{y}\right)+\lambda^{2} T_{u}\right] \psi=0,} \\
\left(\partial_{x}+T_{z}+\lambda T_{u}\right) \psi=0
\end{array}\right.\right.
$$

and the SDYM equations (1) are reduced to the compatibility condition of the Lax pair (18):

$$
\begin{gathered}
\partial_{t} T_{z}-\partial_{x} T_{t}+\left[T_{t}, T_{z}\right]=0, \quad \partial_{x} T_{u}-\left[T_{u}, T_{y}\right]=0 \\
\partial_{t} T_{u}-\partial_{x}\left(T_{z}-T_{y}\right)+\left[T_{t}, T_{u}\right]+\left[T_{z}, T_{y}\right]=0 .
\end{gathered}
$$

To reduce eqs.(19) to the generalized NLS equations, introduced by Fordy and Kulish [27], one should impose the algebraic constraints on the elements of matrices in (19). 
Let us choose in $G L(n, C)$ the subgroups $N$ and $H$ so that $N / H$ be a compact Hermitian symmetric space. Let $\mathcal{N}$ and $\mathcal{H}$ be the Lie algebras of the Lie groups $N$ and $H$. Then $\mathcal{N}=$ $\mathcal{H} \oplus \mathcal{P}$ and $[\mathcal{H}, \mathcal{H}] \subset \mathcal{H},[\mathcal{H}, \mathcal{P}] \subset \mathcal{P},[\mathcal{P}, \mathcal{P}] \subset \mathcal{H}$. A special feature of Hermitian symmetric spaces is the existence of an element $A \in \mathcal{H}$ such that $\mathcal{H}=\{B \in \mathcal{N}:[A, B]=0\}$. The matrix $\operatorname{ad}_{A}$ has only three distinct eigenvalues $0, \pm i$ and $[A, \mathcal{H}]=0,\left[A, X^{ \pm}\right]= \pm i X^{ \pm}$for all $X^{ \pm} \in \mathcal{P}^{ \pm}, \mathcal{P}=\mathcal{P}^{+} \oplus \mathcal{P}^{-}$. Let $e_{ \pm \alpha}$ be a basis of the space $\mathcal{P}^{ \pm}$. Then

$$
\begin{gathered}
{\left[A, e_{ \pm \alpha}\right]= \pm i e_{ \pm \alpha}, \quad\left[e_{\mu},\left[e_{\nu}, e_{-\sigma}\right]\right]=R_{\mu, \nu,-\sigma}^{\alpha} e_{\alpha}} \\
{\left[e_{-\mu},\left[e_{-\nu}, e_{\sigma}\right]\right]=R_{-\mu,-\nu, \sigma}^{-\alpha} e_{-\alpha}, \quad R_{-\mu,-\nu, \sigma}^{-\alpha}=\bar{R}_{\mu, \nu,-\sigma}^{\alpha},}
\end{gathered}
$$

where $R_{\mu, \nu,-\sigma}^{\alpha}$ are components of the curvature tensor defined at the initial point of the symmetric space $N / H$, and $R_{-\mu,-\nu, \sigma}^{-\alpha}$ are complex conjugate to the $R_{\mu, \nu,-\sigma}^{\alpha}$ components.

For the matrices from (17) we choose the following ansatz:

$$
\begin{gathered}
T_{t}=\sum_{\alpha}\left(\phi^{\alpha} e_{\alpha}+\bar{\phi}^{\alpha} e_{-\alpha}\right)+\sum_{\alpha, \beta} \Omega^{\alpha,-\beta}\left[e_{\alpha}, e_{-\beta}\right], \quad T_{u}=A, \\
T_{y}=0, \quad T_{z}=\sum_{\alpha}\left(\psi^{\alpha} e_{\alpha}+\bar{\psi}^{\alpha} e_{-\alpha}\right),
\end{gathered}
$$

where $\phi^{\alpha}, \psi^{\alpha}$ and $\Omega^{\alpha,-\beta}$ are arbitrary complex-valued functions of $t, x$ and the bar over the letter means complex conjugation. Substituting (21) into eqs.(19), we obtain that

$$
\phi^{\alpha}=i \partial_{x} \psi^{\alpha}, \Omega^{\alpha,-\beta}=i\left(\psi^{\alpha} \bar{\psi}^{\beta}+\Omega_{o}^{\alpha,-\beta}\right), \Omega_{o}^{\beta,-\alpha}=\bar{\Omega}^{\alpha,-\beta}=\mathrm{const}
$$

and eqs.(19) are reduced to the generalized NLS equations on the functions $\psi^{\alpha}$ :

$$
i \partial_{t} \psi^{\alpha}+\partial_{x}^{2} \psi^{\alpha}+\sum_{\mu, \nu, \sigma} R_{\mu, \nu,-\sigma}^{\alpha} \psi^{\mu} \psi^{\nu} \bar{\psi}^{\sigma}+\sum_{\mu, \nu, \sigma} R_{\mu, \nu,-\sigma}^{\alpha} \Omega_{o}^{\nu,-\sigma} \psi^{\mu}=0
$$

Notice that the constant components $\Omega_{o}^{\nu,-\sigma}$ can always be chosen so that $\sum_{\nu, \sigma} R_{\mu, \nu,-\sigma}^{\alpha} \Omega_{o}^{\nu,-\sigma}=$ $\omega_{\alpha} \delta_{\mu}^{\alpha}$, where $\omega_{\alpha}$ are real constants [27]. The Lax pair for eqs.(23) can be deduced via substitution of (21) and (22) in (18).

Nonautonomous generalized NLS equation. Now let us consider the two-dimensional Abelian group with the generators $\left\{X_{2}+Y_{2}, P_{u}\right\}$. Then, invariant $A_{\mu}$ and $\Psi$ are given by formulae (12) where $T_{\mu}$ and $\psi$ do not depend on $u$. The reduced Lax pair and SDYM equations have the form

$$
\begin{aligned}
& {\left[\partial_{t}-\zeta \partial_{\rho}+\frac{1}{\rho} \zeta^{2} \partial_{\zeta}+T_{t}-\zeta T_{y}\right] \psi=0, \quad\left[\partial_{\rho}+\frac{1}{\rho} \zeta \partial_{\zeta}+T_{z}+\zeta T_{u}\right] \psi=0} \\
& \partial_{t} T_{z}-\partial_{\rho} T_{t}+\left[T_{t}, T_{z}\right]=0, \quad \partial_{\rho} T_{u}+\left[T_{y}, T_{u}\right]=0,
\end{aligned}
$$




$$
\partial_{\rho}\left(T_{y}-T_{z}\right)+\frac{1}{\rho}\left(T_{y}-T_{z}\right)+\partial_{t} T_{u}+\left[T_{t}, T_{u}\right]+\left[T_{z}, T_{y}\right]=0 .
$$

For matrices from (24), (25) we choose the ansatz (21) again. Substituting (21) into (25), we obtain that

$$
\begin{gathered}
\phi^{\alpha}=i\left(\partial_{\rho} \psi^{\alpha}+\frac{1}{\rho} \psi^{\alpha}\right), \\
\Omega^{\alpha,-\beta}=i\left(\psi^{\alpha} \bar{\psi}^{\beta}+\Omega_{o}^{\alpha,-\beta}+2 \int \frac{d \rho}{\rho} \psi^{\alpha} \bar{\psi}^{\beta}\right), \Omega_{o}^{\beta,-\alpha}=\bar{\Omega}_{o}^{\alpha,-\beta}=\mathrm{const}
\end{gathered}
$$

and the functions $\psi^{\alpha}$ have to satisfy the nonautonomous generalized NLS equations

$$
\begin{gathered}
i \partial_{t} \psi^{\alpha}+\partial_{\rho}^{2} \psi^{\alpha}+\sum_{\mu, \nu, \sigma} R_{\mu, \nu,-\sigma}^{\alpha} \psi^{\mu} \psi^{\nu} \bar{\psi}^{\sigma}+\sum_{\mu, \nu, \sigma} R_{\mu, \nu,-\sigma}^{\alpha} \Omega_{o}^{\nu,-\sigma} \psi^{\mu}= \\
=-\partial_{\rho}\left(\frac{1}{\rho} \psi^{\alpha}\right)-2 \sum_{\mu, \nu, \sigma} R_{\mu, \nu,-\sigma}^{\alpha} \psi^{\mu} \int \frac{d \rho}{\rho} \psi^{\nu} \bar{\psi}^{\sigma} .
\end{gathered}
$$

The Lax pair for eqs.(27) can be obtained by substitution of (21) and (26) into (24).

Remark. In the case of $N=S U(2)$ and $H=U(1)$, ansatz (21) has the form

$$
T_{t}=\left(\begin{array}{cc}
\Omega & \bar{\phi} \\
\phi & -\Omega
\end{array}\right), T_{u}=\frac{1}{2 i}\left(\begin{array}{cc}
1 & 0 \\
0 & -1
\end{array}\right), T_{y}=0, T_{z}=\sqrt{\kappa}\left(\begin{array}{cc}
0 & \bar{\psi} \\
\psi & 0
\end{array}\right)
$$

where $\Omega, \phi$ and $\psi$ are arbitrary complex-valued functions of $t$ and $\rho$, and $\kappa$ is an arbitrary real constant parameter. Substituting (28) into (25), we obtain that

$$
\Omega=-i \kappa\left(\bar{\psi} \psi-\gamma^{2}\right)-2 i \kappa \int \frac{d \rho}{\rho} \bar{\psi} \psi, \quad \phi=i \sqrt{\kappa}\left(\partial_{\rho} \psi+\frac{1}{\rho} \psi\right), \quad \gamma=\text { const },
$$

and the function $\psi$ has to satisfy the equation

$$
i \partial_{t} \psi+\partial_{\rho}^{2} \psi-2 \kappa\left(\bar{\psi} \psi-\gamma^{2}\right) \psi=-\partial_{\rho}\left(\frac{1}{\rho} \psi\right)+4 \kappa \psi \int \frac{d \rho}{\rho} \bar{\psi} \psi
$$

The Lax pair for eqs.(30) can be obtained by substitution of (28) and (29) into (24).

The nonautonomous NLS equation (30) has been considered in the paper [26]. When $\kappa=-1$ and $\gamma^{2}=0$, this equation is gauge equivalent to the equation of the Heisenberg ferromagnet in axial geometry. By change of variables $t, \rho$ and $\psi$, eq.(30) can be transformed to the equation, which has been introduced and integrated in [28]. Thus, the nonautonomous NLS equation is shown to be the reduction of the SDYM equations.

Korteweg-de Vries equation [5,6]. Now, considering the generators $\left\{P_{y}-P_{z}, P_{u}\right\}$, the Lax pair (18) and the compatibility conditions (19), we choose the matrices from (19) in the form of the following $2 \times 2$ matrices

$$
T_{t}=\left(\begin{array}{cc}
a & b \\
c & -a
\end{array}\right), T_{u}=\left(\begin{array}{cc}
0 & 0 \\
-1 & 0
\end{array}\right), T_{y}=\left(\begin{array}{cc}
0 & 0 \\
h & 0
\end{array}\right), T_{z}=\left(\begin{array}{ll}
0 & g \\
f & 0
\end{array}\right)
$$


where $a, b, c, f, g$ and $h$ are arbitrary real-valued functions.

Substituting (31a) in (19), we obtain that

$$
a=\frac{1}{4} \partial_{x} f, b=-\frac{1}{2} f, c=\frac{1}{2} f^{2}+\frac{1}{4} \partial_{x}^{2} f, h=\frac{1}{2} f, g=-1,
$$

and the function $f$ has to satisfy the $\mathrm{KdV}$ equation

$$
\partial_{t} f-\frac{3}{2} f \partial_{x} f-\frac{1}{4} \partial_{x}^{3} f=0
$$

The Lax pair for eqs.(32) is obtained after substitution of (31a) and (31b) into (18).

Nonautonomous $K d V$ equation. Now we consider the generators $\left\{X_{2}+Y_{2}, P_{u}\right\}$, Lax pair (24) and its compatibility conditions (25). For matrices from (25) let us choose the ansatz (31a). Substituting (31a) in (25), we obtain that

$$
\begin{gathered}
a=\frac{1}{4} \partial_{\rho} f-\frac{1}{4 \rho} \int \frac{d \rho}{\rho} f, \quad b=-\frac{1}{2 \rho} f-\frac{1}{2 \rho} \int \frac{d \rho}{\rho} f, \quad h=\frac{1}{2} f+\frac{1}{2} \int \frac{d \rho}{\rho} f, \\
c=\frac{1}{4} \rho \partial_{\rho}^{2} f-\frac{1}{4 \rho} f+\frac{1}{2} f^{2}+\left(\frac{1}{4 \rho}+\frac{f}{2}\right) \int \frac{d \rho}{\rho} f, \quad g=-\frac{1}{\rho},
\end{gathered}
$$

and the function $f$ satisfies the equation

$$
\begin{gathered}
\partial_{t} f-\frac{3}{2} f \partial_{\rho} f-\frac{1}{4} \partial_{\rho}^{3}(\rho f)= \\
=\frac{1}{2 \rho^{2}} f+\frac{1}{2 \rho} f^{2}-\frac{1}{4 \rho} \partial_{\rho} f-\frac{1}{2} \partial_{\rho}^{2} f+\left(\frac{1}{2} \partial_{\rho} f-\frac{f}{2 \rho}-\frac{1}{4 \rho^{2}}\right) \int \frac{d \rho}{\rho} f .
\end{gathered}
$$

The Lax pair for eq.(34) is obtained after substitution of (31a) and (33) into the Lax pair (24).

Remark. Nonautonomous KdV equations have been considered in the papers [26, 28]. Equation (34) differs from ones, considered in [26, 28], and it is a new deformation of the KdV equation.

\section{Reductions to integrable dynamical systems}

Periodic Toda lattice with damping. Let us consider the three-dimensional non-Abelian subgroups of $I S O(2,2)$ generated by the vector fields $X_{2}+\beta Y_{2}, P_{y}, P_{z}$, where $\beta \in R, \beta \neq 1$. Notice that the SDYM equations, reduced with respect to the symmetry group with the generators $X_{2}+Y_{2}, P_{y}$ and $P_{z}$, lead to the zero curvature condition $F_{\mu \nu}=0$ and, therefore, they are not interesting. That is why we shall investigate the case $\beta \neq 1$.

Let us introduce the coordinates $\tau, \theta$ by formulae $\tau=\frac{1}{4} \ln (4 t u)^{2}, \theta=\frac{1}{4} \ln \left(\frac{u}{t}\right)^{2}$. Then, the orbit coordinates are $\chi=\frac{2\left(1+\beta^{2}\right)}{(1-\beta)} \theta+\frac{1}{2} \beta \ln (\bar{\lambda} \lambda), y, z$ and the invariant coordinates 
are $\tau, \zeta=\lambda e^{\gamma \theta}$, where $\gamma=2 \beta /(1-\beta)$. The invariant YM potentials and $\Psi$ satisfying eqs.(2c) and (6) are given by

$$
\begin{gathered}
A_{t}=T_{t}(\tau) e^{\theta-\tau}, A_{u}=T_{u}(\tau) e^{-\theta-\tau}, A_{y}=T_{y}(\tau) e^{(1+\gamma)(\theta-\tau)}, A_{z}=T_{z}(\tau) e^{-(1+\gamma)(\theta+\tau)} \\
\Psi=\psi(\tau, \zeta) .
\end{gathered}
$$

Substituting (35) into the linear system (2), changing the variables and using (6), we obtain the following reduced Lax pair:

$$
\left[\partial_{\tau}-\gamma \zeta \partial_{\zeta}+T_{t}-\zeta e^{-\gamma \tau} T_{y}\right] \psi=0, \quad\left[\zeta \partial_{\tau}+\gamma \zeta^{2} \partial_{\zeta}+e^{-\gamma \tau} T_{z}+\zeta T_{u}\right] \psi=0,
$$

Using the compatibility condition (14) for the Lax pair (36), we obtain the following reduced SDYM equations:

$$
\begin{aligned}
& \frac{d}{d \tau} T_{y}+\left[T_{u}, T_{y}\right]=0, \quad \frac{d}{d \tau} T_{z}+\left[T_{t}, T_{z}\right]=0, \\
& \frac{d}{d \tau}\left(T_{u}-T_{t}\right)+\left[T_{t}, T_{u}\right]+e^{-2 \gamma \tau}\left[T_{z}, T_{y}\right]=0 .
\end{aligned}
$$

The equations of the periodic Toda lattice with damping are derived via the algebraic reduction of eqs.(37). Let us choose for $T_{t}, T_{u}, T_{y}, T_{z} \in g l(n, C)$ the following (algebraic) ansatz:

$$
T_{t}=-T_{u}=\left(\begin{array}{cccc}
p_{1} & 0 & \ldots & 0 \\
0 & p_{2} & \ddots & \vdots \\
\vdots & \ddots & \ddots & 0 \\
0 & \ldots & 0 & p_{n}
\end{array}\right), \quad T_{y}=T_{z}^{T}=2\left(\begin{array}{ccccc}
0 & a_{1} & 0 & \ldots & 0 \\
0 & 0 & a_{2} & \ddots & \vdots \\
\vdots & \ddots & \ddots & \ddots & 0 \\
0 & 0 & \ldots & 0 & a_{n-1} \\
a_{n} & 0 & \ldots & 0 & 0
\end{array}\right),
$$

where $a_{\alpha}=\exp \left(q_{\alpha}-q_{\alpha+1}\right)$ and the superscript ${ }^{T}$ means matrix transpose. Then, after substitution of (38) into eqs.(37), we obtain

$$
\frac{d}{d \tau} q_{\alpha}=p_{\alpha}, \quad \frac{d}{d \tau} p_{\alpha}=2 \exp (-2 \gamma \tau)\left\{\exp \left[2\left(q_{\alpha-1}-q_{\alpha}\right)\right]-\exp \left[2\left(q_{\alpha}-q_{\alpha+1}\right)\right]\right\}
$$

When $\gamma=0$, the latter equations coincide with the standard periodic Toda lattice equations. If $\gamma \neq 0$, then using the variable $\varphi=\exp (-\gamma \tau)$, we obtain the equations of the Toda lattice with damping:

$$
\frac{d^{2}}{d \varphi^{2}} q_{\alpha}+\frac{1}{\varphi} \frac{d}{d \varphi} q_{\alpha}=\frac{2}{\gamma^{2}}\left\{\exp \left[2\left(q_{\alpha-1}-q_{\alpha}\right)\right]-\exp \left[2\left(q_{\alpha}-q_{\alpha+1}\right)\right]\right\} .
$$

The corresponding Lax pair is obtained by inserting (38) in (36). 
Integrable Hamiltonian systems with quartic potentials. We are still considering the generators $X_{2}+\beta Y_{2}, P_{y}, P_{z}$, the Lax pair (36) and the compatibility conditions (37). Let us choose in $G L(n, C)$ the subgroups $N$ and $H$ in such a way that $N / H$ be the Hermitian symmetric space (for definitions and notation see Sec.5).

For the matrices from (37) we choose the following ansatz:

$$
\begin{gathered}
T_{t}=0, \quad T_{u}=i \sum_{\alpha} q^{\alpha}\left(e_{\alpha}+e_{-\alpha}\right), \\
T_{y}=\sum_{\alpha} r^{\alpha}\left(e_{\alpha}-e_{-\alpha}\right)+\sum_{\alpha, \sigma} \Omega^{\alpha,-\sigma}\left[e_{\alpha}, e_{-\sigma}\right], \quad T_{z}=A,
\end{gathered}
$$

where $q^{\alpha}, r^{\alpha}$ and $\Omega^{\alpha,-\sigma}$ are arbitrary real-valued functions of $\tau$.

Substituting (41) in (37) we obtain that

$$
r^{\alpha}=-e^{2 \gamma \tau} \frac{d q^{\alpha}}{d \tau}, \Omega^{\alpha,-\sigma}=i \Omega_{o}^{\alpha,-\sigma}-i e^{2 \gamma \tau} q^{\alpha} q^{\sigma}+2 i \gamma \int d \tau e^{2 \gamma \tau} q^{\alpha} q^{\sigma}, \Omega_{o}^{\alpha,-\sigma}=\text { const }
$$

and eqs.(37) are reduced to the equations

$$
\begin{gathered}
\frac{d^{2}}{d \tau^{2}} q^{\alpha}-\sum_{\mu, \nu, \sigma} R_{\mu, \nu,-\sigma}^{\alpha} q^{\mu} q^{\nu} q^{\sigma}+\sum_{\mu, \nu, \sigma} R_{\mu, \nu,-\sigma}^{\alpha} \Omega_{o}^{\nu,-\sigma} q^{\mu}= \\
=-2 \gamma\left(\frac{d q^{\alpha}}{d \tau}+e^{-2 \gamma \tau} \sum_{\mu, \nu, \sigma} R_{\mu, \nu,-\sigma}^{\alpha} q^{\mu} \int d \tau e^{2 \gamma \tau} q^{\nu} q^{\sigma}\right)+\left(1-e^{-2 \gamma \tau}\right) \sum_{\mu, \nu, \sigma} R_{\mu, \nu,-\sigma}^{\alpha} \Omega_{o}^{\nu,-\sigma} q^{\mu} .
\end{gathered}
$$

Notice that $\Omega_{o}^{\alpha,-\sigma}$ may always be chosen so that $\sum_{\nu, \sigma} R_{\mu, \nu,-\sigma}^{\alpha} \Omega_{o}^{\nu,-\sigma}=\omega_{\mu} \delta_{\mu}^{\alpha}$, where $\omega_{\mu}=$ const.

When $\gamma=0$, eqs.(43) coincide with the equations of motion in quartic potentials, considered in [29]. Equations of the Garnier system are the particular case of eqs.(43), corresponding to $\gamma=0, N=S U(n), H=S(U(1) \times U(n-1))$. The Lax pair for eqs.(43) can be obtained by inserting (41) and (42) into (36).

Euler-Arnold equations and their deformations. Now let us consider the three-dimensional non-Abelian symmetry group with the generators $\alpha X_{2}+\beta Y_{2}, P_{u}, P_{y}$, where $\alpha, \beta \in$ $R, \alpha^{2}-\beta^{2}=1$. Let us introduce the coordinates $\tau=\frac{1}{2}(\alpha-\beta) \ln z^{2}+\frac{1}{2}(\alpha+\beta) \ln t^{2}, \theta=$ $\frac{1}{2}(\alpha-\beta) \ln z^{2}-\frac{1}{2}(\alpha+\beta) \ln t^{2}$. In this case the orbits are parametrized by the coordinates $\chi=\theta-\frac{1}{2} \beta \ln (\bar{\lambda} \lambda), u, y$ and the invariant coordinates are $\tau, \zeta=\lambda e^{\beta \theta}$. Solving eqs. (6) and $(2 \mathrm{c})$, we obtain the following formulae for the invariant YM potentials and for the vector-function $\Psi$

$$
A_{t}=(\alpha+\beta) \exp \left(\frac{\theta-\tau}{2(\alpha+\beta)}\right) T_{t}(\tau), A_{u}=(\alpha-\beta) \exp \left(-\frac{\tau}{2(\alpha-\beta)}-\frac{\theta}{2(\alpha+\beta)}\right) T_{u}(\tau)
$$




$$
\begin{gathered}
A_{y}=(\alpha+\beta) \exp \left(-\frac{\tau}{2(\alpha+\beta)}+\frac{\theta}{2(\alpha-\beta)}\right) T_{y}(\tau), A_{z}=(\alpha-\beta) \exp \left(\frac{-\theta-\tau}{2(\alpha-\beta)}\right) T_{z}(\tau), \\
\Psi=\psi(\tau, \zeta) .
\end{gathered}
$$

Substitute (44) into the linear system (2) and express the derivatives in (2) via the new coordinates. Then, after using the conditions of invariance of $\psi$, the linear system (2) is reduced to the following one:

$$
\left(\partial_{\tau}-\beta \zeta \partial_{\zeta}+T_{t}-\zeta T_{y}\right) \psi=0, \quad\left(\partial_{\tau}+\beta \zeta \partial_{\zeta}+T_{z}+\zeta T_{u}\right) \psi=0
$$

If we put $N_{t}=\frac{1}{2}\left(T_{z}+T_{t}\right), N_{u}=\frac{1}{2}\left(T_{y}+T_{u}\right), N_{y}=\frac{1}{2}\left(T_{y}-T_{u}\right), N_{z}=\frac{1}{2}\left(T_{z}-T_{t}\right)$, then we can rewrite the Lax pair (45) in the form

$$
\left(\partial_{\tau}+N_{t}-\zeta N_{y}\right) \psi=0, \quad\left(\beta \zeta \partial_{\zeta}+N_{z}+\zeta N_{u}\right) \psi=0
$$

The compatibility conditions of the Lax pair (46) are

$$
\begin{gathered}
\partial_{\tau} N_{u}+\beta N_{y}+\left[N_{t}, N_{u}\right]+\left[N_{z}, N_{y}\right]=0, \quad\left[N_{u}, N_{y}\right]=0 \\
\partial_{\tau} N_{z}+\left[N_{t}, N_{z}\right]=0 .
\end{gathered}
$$

Let us choose $N_{t}, N_{z}$ to be antisymmetric $n \times n$ matrices and $N_{u}, N_{y}$ to be diagonal matrices satisfying the equation $\partial_{\tau} N_{u}+\beta N_{y}=0$. We choose the solution of this equation in the following form: $N_{u}=\mathcal{A}-\beta \tau N_{y}$, where $\mathcal{A}=\operatorname{diag}\left(a_{1}, \ldots, a_{n}\right)$ and $N_{y}=\operatorname{diag}\left(b_{1}, \ldots, b_{n}\right)$ are constant diagonal matrices with $a_{i} \neq a_{j}, b_{i} \neq b_{j}$ and $a_{i} \neq b_{j}$ when $i \neq j$. Solution of eqs.(47a) may be written in the form

$$
N_{u}=\mathcal{A}-\beta \tau N_{y}, \quad\left(N_{t}\right)_{i j}=\lambda_{i j} M_{i j}, \quad\left(N_{z}\right)_{i j}=\left(\beta \tau \lambda_{i j}-1\right) M_{i j}
$$

where $\lambda_{i j}=\left(b_{i}-b_{j}\right) /\left(a_{i}-a_{j}\right), M=\left(M_{i j}\right)=\left(-M_{j i}\right)$ is an arbitrary antisymmetric matrix and $\left(N_{t}\right)_{i j},\left(N_{z}\right)_{i j}$ are components of the matrices $N_{t}, N_{z}$.

After substitution of (48), eqs.(47b) can be written in the form

$$
\frac{d}{d \tau}\left[\left(1-\beta \tau \lambda_{i j}\right) M_{i j}\right]=\sum_{k}\left(\lambda_{k j}-\lambda_{i k}\right) M_{i k} M_{k j} .
$$

When $\beta=0$, these equations coincide with the Euler-Arnold equations describing the rotation of the $n$-dimensional rigid body [30]. One may also rewrite eqs.(49) in the form

$$
\frac{d}{d \tau} W_{i j}=\sum_{k}\left(\Lambda_{k j}-\Lambda_{i k}\right) W_{i k} W_{k j},
$$

where $W_{i j}=\left(1-\beta \tau \lambda_{i j}\right) M_{i j}$ and $\Lambda_{i j}(\tau)=\lambda_{i j} /\left(1-\beta \tau \lambda_{i j}\right)$. 
Generalized Calogero-Moser system. Here we consider the same symmetry group with the generators $\alpha X_{2}+\beta Y_{2}, P_{u}, P_{y}\left(\alpha, \beta \in R, \alpha^{2}-\beta^{2}=1\right)$, the Lax pair (46) and the compatibility conditions (47). Now for the matrices $N_{t}, N_{u}, N_{y}, N_{z} \in g l(n, C)$ we choose the following algebraic ansatz:

$$
\begin{gathered}
N_{t}=-i \sum_{\substack{j, k=1 \\
j \neq k}}^{n} \frac{f_{j}^{+} f_{k}}{\left(q_{j}-q_{k}\right)^{2}} e_{j k}-2 N_{z}, \quad N_{u}=-i \sum_{j, k=1}^{n} f_{j}^{+} f_{k} e_{j k}, \quad N_{y}=2 N_{u}, \\
N_{z}=h\left\{\sum_{k=1}^{n} p_{k} e_{k k}+i \sum_{\substack{j, k=1 \\
j \neq k}}^{n} \frac{f_{j}^{+} f_{k}}{q_{j}-q_{k}} e_{j k}\right\} .
\end{gathered}
$$

Here $p_{j}, q_{j}$ and $h$ are the real-valued functions of $\tau$, the vector-functions $f_{j}(\tau)$ belong to $N$-dimensional vector space $C^{N}, f_{j}^{+}$are canonical conjugate to $f_{j}: f_{j}^{+} f_{j}=\sum_{a=1}^{N} \bar{f}_{j}^{a} f_{j}^{a}=1$, and $\left(e_{j k}\right)_{m n}=\delta_{j m} \delta_{k n}$ are the generators of the group $S L(n, C):\left[e_{j k}, e_{l m}\right]=\delta_{k l} e_{j m}-\delta_{m j} e_{l k}$.

Substituting (51) into (47), we obtain that $h=\exp (2 \beta \tau)$ and eqs.(47) are reduced to the following system of equations:

$$
\begin{gathered}
\frac{d}{d \tau} q_{j}=p_{j}, \frac{d}{d \tau} p_{j}+2 \beta p_{j}=2 \sum_{\substack{k=1 \\
k \neq j}}^{n} \frac{\left(f_{j}^{+} f_{k}\right)\left(f_{k}^{+} f_{j}\right)}{\left(q_{j}-q_{k}\right)^{3}}, \\
\frac{d}{d \tau} f_{j}+\beta f_{j}=-i \sum_{\substack{k=1 \\
k \neq j}}^{n} \frac{f_{k}\left(f_{k}^{+} f_{j}\right)}{\left(q_{j}-q_{k}\right)^{2}}, \quad \frac{d}{d \tau} f_{j}^{+}+\beta f_{j}^{+}=i \sum_{\substack{k=1 \\
k \neq j}}^{n} \frac{\left(f_{j}^{+} f_{k}\right) f_{k}^{+}}{\left(q_{j}-q_{k}\right)^{2}} .
\end{gathered}
$$

The Lax pair for eqs.(52) can be obtained by substituting (51) into (46). When $\beta=0$, eqs.(52) coincide with those of the generalized Calogero-Moser system introduced in [31] (see also [32] and references therein). It may be shown that by change of variables $\tau, p_{j}$ and $f_{j}$, eqs.(52) can be transformed to the standard equations with $\beta=0$.

Euler-Calogero-Moser system. Considering the same symmetry group with the generators $\alpha X_{2}+\beta Y_{2}, P_{u}, P_{y} \quad\left(\alpha, \beta \in R, \alpha^{2}-\beta^{2}=1\right)$, the Lax pair (46) and its compatibility conditions (47), for matrices in (46), (47) we choose now the following ansatz:

$$
\begin{gathered}
N_{t}=\sum_{\substack{j, k=1 \\
j \neq k}}^{n} \frac{h_{j k}}{\left(q_{j}-q_{k}\right)^{2}} e_{j k}-2 N_{z}, \quad N_{u}=-\sum_{j, k=1}^{n} h_{j k} e_{j k}, \quad N_{y}=2 N_{u}, \\
N_{z}=\exp (2 \beta \tau)\left\{\sum_{k} p_{k} e_{k k}-\sum_{\substack{j, k=1 \\
j \neq k}}^{n} \frac{h_{j k}}{q_{j}-q_{k}} e_{j k}\right\},
\end{gathered}
$$

where $p_{j}, q_{j}$ and $h_{i j}=-h_{j i}$ are the real-valued functions of $\tau$, and $e_{j k}$ are the generators of the group $S L(n, R)$. 
Substituting (53) into eqs.(47) yields

$$
\begin{gathered}
\frac{d}{d \tau} q_{j}=p_{j}, \frac{d}{d \tau} p_{j}+2 \beta p_{j}=2 \sum_{\substack{k=1 \\
k \neq j}}^{n} \frac{h_{j k} h_{j k}}{\left(q_{j}-q_{k}\right)^{3}}, \\
\frac{d}{d \tau} h_{j k}+2 \beta h_{j k}=\sum_{\substack{m=1 \\
m \neq j, k}}^{n} h_{j m} h_{m k}\left[\frac{1}{\left(q_{k}-q_{m}\right)^{2}}-\frac{1}{\left(q_{j}-q_{m}\right)^{2}}\right] .
\end{gathered}
$$

The Lax pair for eqs.(54) has the form (46) with the matrices $N_{t}, N_{u}, N_{y}, N_{z}$ from (53). When $\beta=0$, eqs.(54) coincide with those of the Euler-Calogero-Moser system introduced in the paper [33] (see also the discussion of this integrable system in [32]). It may be shown that by change of variables $\tau, p_{j}$ and $h_{i j}$, eqs. (54) can be transformed to the standard equations with $\beta=0$.

\section{Conclusion}

In this paper, we have introduced six new systems of nonlinear integrable differential equations. The Lax pairs for all of these systems contain derivatives of the form $\zeta \frac{\partial}{\partial \zeta}$ with respect to the spectral parameter $\zeta$. The differential operator $\zeta \frac{\partial}{\partial \zeta}$ corresponds to the extention of the loop algebra associated with the Lie algebra of the gauge group, and this extention is important in the standard approach to the integrable equations in $(1+1)$ dimensions [34]. The dressing method for the Lax pairs containing the additional term $\zeta \partial_{\zeta}$ has been developed by Belinsky and Zakharov [25]. Namely, if one chooses a "seed solution" of an integrable system and constructs the corresponding solution $\psi_{0}$ of the linear system, then the ansatz for iteration is $\psi_{n}=\left(I+\frac{R_{n}}{\zeta-\mu_{n}}\right) \psi_{n-1}$, where the matrices $R_{n}$ are independent of $\zeta$, and $\mu_{n}$ are functions of the coordinates and do not depend on $\zeta$ (moving poles). For more detailed discussions see, e.g., [25, 26, 34].

\section{Acknowledgements}

One of us (A.D.P.) thanks for support and hospitality the Max-Planck-Institut für Physik, München, where part of this work was done. This work was supported by the International Science Foundation (grant \# NK8300), by the Russian Foundation for Fundamental Research (grant \# 95-01-00027) and by the Heisenberg-Landau Program. 


\section{References}

1. P.J. Olver, Applications of Lie Groups to Differential Equations (Springer-Verlag, New York, 1986); P.Winternitz, in: Partially Integrable Evolution Equations in Physics, eds. R.Conte and N.Boccara, NATO ASI Ser. C, v.310, p.515 (Kluwer Academic Publ., Dordrecht, 1990).

2. P.Forgács and N.S.Manton, Commun.Math.Phys. 72 (1980) 15; J.Harnad, S.Snider and L.Vinet, J.Math.Phys. 21 (1980) 2719; R.Jackiw and N.S.Manton, Ann.Phys. 127 (1980) 257.

3. M.Legaré and A.D.Popov, Phys.Lett. A 198 (1995) 195; JETP Lett. 59 (1994) 883; T.A.Ivanova and A.D.Popov, Teor.Mat.Fiz. 102 (1995) 384; JETP Lett. 61 (1995) 150 .

4. R.S.Ward, Phil.Trans.R.Soc.Lond. A315 (1985) 451; Lect.Notes in Phys. 280 (1987) 106; R.S.Ward, in: Twistors in Mathematics and Physics, eds. T.N. Bailey and R.J. Baston, London Math. Society Lect. Note Ser., v. 156, p.246 (Cambridge University Press, Cambridge, 1990).

5. L.J.Mason and G.A.J.Sparling, Phys.Lett.A 137 (1989) 29; J.Geom.and Phys. 8 (1992) 243.

6. I.Bakas and D.A.Depireux, Mod.Phys.Lett.A 6 (1991) 399; 1561.

7. M.J.Ablowitz, S.Chakravarty and P.A.Clarkson, Phys.Rev.Lett. 65 (1990) 1085;

S.Chakravarty and M.J.Ablowitz, in: Painlevé Transcendents, their Asymptotics and Physical Applications, eds. D.Levi and P.Winternitz, NATO ASI Ser. B, v. 278, p.331 (Plenum Press, New York, 1992).

8. S.Chakravarty, S.Kent and E.T.Newman, J.Math.Phys. 31 (1990) 2253; 33 (1992) 382; I.A.B.Strachan, Phys.Lett.A 154 (1991) 123; T.A.Ivanova and A.D.Popov, Lett. Math. Phys. 23 (1991) 29.

9. T.A.Ivanova and A.D.Popov, Phys.Lett.A 170 (1992) 293.

10. R.S.Ward, Gen.Rel.Grav. 15 (1983) 105; N.M.J.Woodhouse, Class. Quantum Grav. 4 (1987) 799; 6 (1989) 933; N.M.J.Woodhouse and L.J.Mason, Nonlinearity 1 (1988) 73; J.Fletcher and N.M.J.Woodhouse, in: Twistors in Mathematics and Physics, eds. 
T.N. Bailey, R.J. Baston, London Math. Society Lect. Note Ser., v. 156, p. 260 (Cambridge University Press, Cambridge, 1990).

11. L.J.Mason and N.M.J.Woodhouse, Nonlinearity 6 (1993) 569.

12. J.Tafel, J.Math.Phys. 34 (1993) 1892.

13. M.Kovalyov, M.Legaré and L.Gagnon, J.Math.Phys. 34 (1993) 3245.

14. M.J.Ablowitz and P.A.Clarkson, Solitons, Nonlinear Evolution Equations and Inverse Scattering (Cambrige University Press, Cambridge, 1991).

15. M.F.Atiyah, Classical Geometry of Yang-Mills Fields (Scuola Normale Superiore, Pisa, 1979); M.Atiyah and N.Hitchin, The Geometry and Dynamics of Magnetic Monopoles (Princeton University Press, Princeton, 1988); R.S.Ward and R.O.Wells Jr., Twistor Geometry and Field Theory (Cambridge University Press, Cambridge, 1990).

16. R.S.Ward, J.Math.Phys. 29 (1988) 386; Nonlinearity 1 (1988) 671; J.Math.Phys. 30 (1989) 2246; Commun.Math.Phys. 128 (1990) 319.

17. H.Ooguri and C.Vafa, Mod.Phys.Lett. A5 (1990) 1389; Nucl. Phys. B361 (1991) 469; B367 (1991) 83; W.Siegel, Phys.Rev.Lett. 69 (1992) 1493; Phys.Rev. D46 (1992) 3235; D47 (1993) 2504; D47 (1993) 2512.

18. A.Parkes, Nucl.Phys. B376 (1992) 279; S.V.Ketov, H.Nishino and S.J.Gates Jr., Phys. Lett. B307 (1993) 331; Nucl.Phys. B393 (1993) 149.

19. N.Berkovits and C.Vafa, Mod.Phys.Lett. A9 (1994) 653; Nucl.Phys. B433 (1995) 123.

20. N.Berkovits, Super-Poincaré invariant superstring field theory, hep-th/9503009; New spacetime-supersymmetric methods for the superstring, hep-th/9506036.

21. A.A.Belavin and V.E.Zakharov, Phys.Lett. B73 (1978) 53.

22. R.S.Ward, Phys.Lett. A61 (1977) 81.

23. A.Lichnerowicz, Géométrie des groupes de transformations (Dunod, Paris, 1958); S.Kobayashi, Transformation groups in differential geometry (Springer-Verlag, Berlin, $1972)$. 
24. S.V.Manakov and V.E.Zakharov, Lett.Math.Phys. 5 (1981) 247.

25. V.A.Belinsky and V.E.Zakharov, Zh.Eksp.Teor.Fiz. 75 (1978) 1953; 77 (1979) 3.

26. S.P.Burtsev, V.E.Zakharov and A.V.Mikhailov, Teor.Mat.Fiz. 70 (1987) 323.

27. A.P.Fordy and P.P.Kulish, Commun.Math.Phys. 89 (1983) 427.

28. F.Calogero and A.Degasperis, Commun.Math.Phys. 63 (1978) 155.

29. A.Fordy, S.Wojciechowski and I.Marshall, Phys.Lett. 113A (1986) 395.

30. A.T.Fomenko and V.V.Trofimov, Integrable systems on Lie algebras and symmetric spaces (Gordon and Breach, New York, 1988).

31. J.Gibbons and Th.Hermsen, Physica 11D (1984) 337.

32. E.Billey, J.Avan and O.Babelon, Phys.Lett. A186 (1994) 114; A188 (1994) 263.

33. S.Wojciechowski, Phys.Lett. A111 (1985) 101.

34. A.C.Newell, Solitons in Mathematics and Physics (SIAM, Philadelphia, 1985). 